\title{
Influence of spacer device on drug delivery to young children with asthma
}

Department of Paediatrics, Kolding Hospital, DK-6000 Kolding, Denmark

L Agertoft

$S$ Pedersen

Correspondence to: Dr Agertoft.

Accepted 8 June 1994

Lone Agertoft, Søren Pedersen

\begin{abstract}
The budesonide dose delivered to the patient from three different spacer devices (Nebuhaler $=750 \mathrm{ml}$, Aerochamber $=140 \mathrm{ml}$, and Babyspacer $=260$ $\mathrm{ml}$ ) was assessed by measuring the budesonide dose deposited on a filter inserted between the spacer outlet and the mouth of the patient. Twenty children aged 10-25 months were given a single dose of $200 \mu \mathrm{g}$ budesonide from each spacer device in a randomised crossover study. All spacers had a facemask attached and a one way valve system. The children breathed through the inhalation system for 30 seconds. Furthermore, the minute ventilation of the children through a tightly fitting facemask was measured.

The filter dose of budesonide was significantly lower after Aerochamber treatment (39.4 $\mu \mathrm{g}$, range $19-67 \mu \mathrm{g})$ than after Nebuhaler $(53.5 \mu \mathrm{g}$, range 34-88 $\mu \mathrm{g})$ and Babyspacer $(55.5 \mu \mathrm{g}$, range 39-76 $\mu \mathrm{g})$ treatment. The minute ventilation of the children varied from $1.4 \mathrm{l} / \mathrm{min}$ to $7.0 \mathrm{l} / \mathrm{min}$ (mean $5.0 \mathrm{l} / \mathrm{min}$ ). This was sufficient to empty all spacers within the 30 seconds of inhalation. It is concluded that spacer volume does not seem to be so important for children aged 10-25 months as long as spacers with a volume lower than $750 \mathrm{ml}$ are used.
\end{abstract}

(Arch Dis Child 1994; 71: 217-220)

Effective inhalation treatment in young children is difficult. At present nebulisers are very popular in this age group, but these inhalers are bulky, expensive, time consuming, and ineffective delivery systems which require electrical supply. For these reasons spacer devices with valve systems are a theoretically more attractive alternative. However, knowledge about the dose that young patients are inhaling from these devices is sparse as is the information about the importance of differences in design between the various spacer systems. This information is important for the clinician prescribing these inhalers to young children. The aim of the present study was to measure the dose of budesonide that young children inhale from three different spacer devices each with a facemask: a large volume spacer (Nebuhaler $=750 \mathrm{ml}$, Astra); a tube shaped low volume spacer (Aerochamber $=140 \mathrm{ml}$, Boehringer Ingelheim); and a round low volume spacer (Babyspacer $=260$ $\mathrm{ml}$, Astra). Apart from differences in volume and shape the various spacers also had different valve systems.
Patients and methods

Twenty children with asthma who were assessed to require continuous treatment with inhaled corticosteroids participated in the study. Their age varied from 10 to 25 months (mean 16 months). None of the children had tried inhaled treatment with a metered dose inhaler and a spacer before. Written informed consent was obtained from all parents and the study was approved by the local ethics committee.

The design was a controlled, randomised crossover study. Each child inhaled a single puff of $200 \mu \mathrm{g}$ budesonide from the three devices in randomised sequence. The Aerochamber was a model without an expiratory valve. Each inhalation was separated by 20 minutes from the previous inhalation. The children were asymptomatic on the day of treatment. The same person administered all the inhalations.

The inhalation technique was the same for all delivery systems. After an initial demonstration of the correct use of the device by the nurse a dose of budesonide was actuated into the spacer. The facemask was fitted tightly around the child's nose and mouth by the nurse during actuation and the child was sitting with its mother. The child breathed in and out through the system for 30 seconds after which the inhalation device was removed. Each child was only given one try with each inhaler. All devices were tilted upwards at an angle of approximately $45^{\circ}$ during the inhalation.

The dose to the patient was defined as the dose deposited on a filter inserted between the lips of the patient and the inhalation system. The filter system added an extra dead space of $45 \mathrm{ml}$. The filter was a Marquest viral filter that has been shown to collect virtually $100 \%$ of the dose inhaled through it. ${ }^{1}$ This technique of dose assessment has previously been used to assess the dose to the patient from nebulisers. ${ }^{1}$

The same conventional metered dose budesonide inhaler delivering $200 \mu \mathrm{g}$ was used for all administrations. One hour before the first inhalation the spacers were primed by firing five budesonide doses into the spacer.

After each treatment the filter was sealed with a tightly fitting plastic cover. After completion of the study the budesonide was extracted from the filters by washing with spectrometrically pure ethanol and the budesonide concentration and dose were determined by reversed phase high performance liquid chromatography. The laboratory was blind with respect to the inhaler used.

Twenty minutes after the third administration each child breathed normally for two 
Dose difference: Babyspacer-Aerochamber

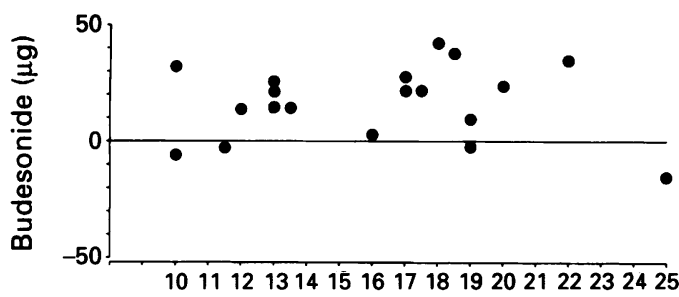

Dose difference: Babyspacer-Nebuhaler

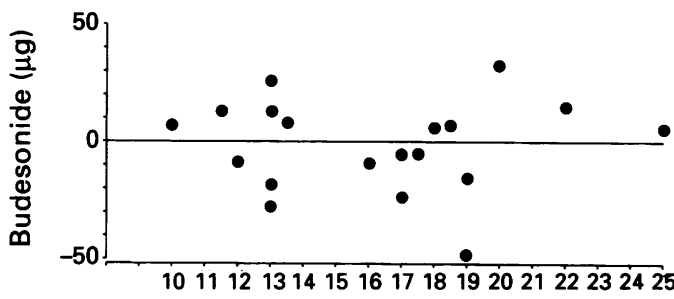

Dose difference: Nebuhaler-Aerochamber

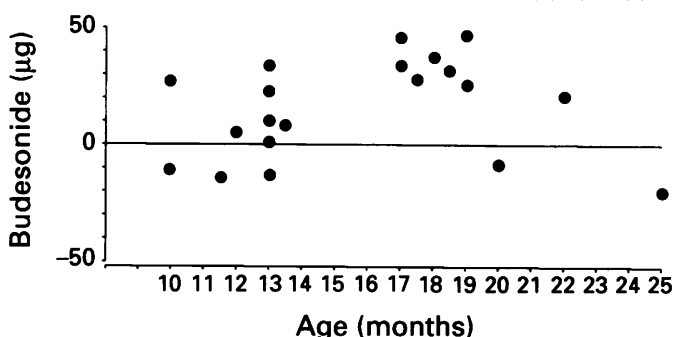

Individual differences in inhaled budesonide dose after administration of $200 \mu \mathrm{g}$ from three different spacer devices (Aerochamber, Nebuhaler, and Babyspacer) in 20 children aged 10-25 months.

minutes through a facemask with a one way valve connected to a Magtrak II respiratory monitor and sensor (Ferraris Medical, UK). This allowed measurement of the number of breaths per minute and tidal volume of the child during breathing through a facemask.

After the study an in vitro test was performed to evaluate how much budesonide impacted on the filter after actuation alone, before any inhalation was performed: one dose of budesonide was released into each spacer and after two seconds the dose was 'inhaled' with a continuous flow rate of $30 \mathrm{l} / \mathrm{min}$ for 10 seconds. This procedure was performed twice for each inhaler, one with the filter attached during actuation and one when the filter was attached immediately after the actuation. These measurements were not performed on the same spacers as used in the study.

\section{STATISTICS}

Friedman's test was used to evaluate overall differences and period effects between the three inhalers. If differences were found individual comparisons were made by Wilcoxon rank sum test; $p<0.05$ was considered statistically significant.

\section{Results}

All 20 children completed the three treatments and the measurement of minute ventilation. Six received Nebuhaler as first treatment, seven the Aerochamber, and seven the Babyspacer. There was no difference between the budesonide dose deposited on the inspiratory filter after treatment 1 , treatment 2 , and treatment $3(49.8 \mu \mathrm{g}$, $47 \cdot 2 \mu \mathrm{g}$, and $51.2 \mu \mathrm{g}$, respectively), indicating that increasing experience of the child with the number of inhalations did not influence the result. The inhalation device was accepted by the child without any problems in about half the inhalations. The remaining inhalations were complicated by the child's crying or struggling during the inhalation. The occurrence of these problems was the same for all three spacers and it did not significantly influence the filter dose.

Comparisons of the individual budesonide filter doses after the three treatments are shown in the figure. The mean (SD) filter dose after Aerochamber treatment was significantly lower (39.4 (13.6) $\mu \mathrm{g}$, range 19-67 $\mu \mathrm{g}$ ) than the filter dose after the other treatments $(53.5(10.4) \mu \mathrm{g}$, range 34-88 $\mu \mathrm{g}$ for the Nebuhaler and 55.5 $(10 \cdot 4) \mu \mathrm{g}$, range 39-76 $\mu \mathrm{g}$ for the Babyspacer) $(\mathrm{p}<0.01)$. There was no statistically significant difference between the filter dose after Nebuhaler and Babyspacer treatment. The age of the child did not influence the result.

The in vitro testing showed that for the Nebuhaler and Babyspacer the filter dose was the same when budesonide was actuated into the spacer with the filter attached during actuation $(61 \mu \mathrm{g}$ for the Nebuhaler and $58 \mu \mathrm{g}$ for the Babyspacer) as when the filter was attached immediately after the actuation (60 $\mu \mathrm{g}$ for the Nebuhaler and $57 \mu \mathrm{g}$ for the Babyspacer). For the Aerochamber $44 \mu \mathrm{g}$ was deposited on the filter when attached during actuation and $31 \mu \mathrm{g}$ when it was not attached. Thus it seemed that initial leakage through the valve could account for about $30 \%$ of the Aerochamber filter dose in our study.

Tidal volume varied from $60-260 \mathrm{ml}$ (mean $=192 \mathrm{ml}$ ) and the number of breaths per minute from 16-44 (mean $26 \cdot 2$ ) resulting in a mean minute ventilation of $5.0 \mathrm{l} / \mathrm{min}$ (range $1 \cdot 4-7 \cdot 1 \mathrm{l} / \mathrm{min})$.

\section{Discussion}

When the results of the study are evaluated it should be realised that the dose on the filter is not equivalent to the dose of drug deposited in the intrapulmonary airways, as a $10 \mu \mathrm{m}$ drug particle contains approximately 40 times as much drug as a $3 \mu \mathrm{m}$ drug particle. However, though direct comparisons have not been made, the particle size distribution of budesonide particles leaving the three inhalers seems to be quite similar. If this is the case it can be assumed that the amount of drug deposited on the inspiratory filter will parallel the amount of drug reaching the intrapulmonary airways.

The filter dose gives an accurate estimate of the fraction of the nominal dose that is actually delivered to the patient and the filter method has been used earlier for such assessments for nebulisers. ${ }^{1}$ This information is important when the safety of inhaled corticosteroids is evaluated. In pharmacokinetic studies ${ }^{2}$ and filter studies in schoolchildren (data on file) the filter dose after inhalation of $200 \mu \mathrm{g}$ budesonide from a Nebuhaler is around $90 \mu \mathrm{g}$ and 
the bioavailability of drug around $25 \%$ of the nominal dose. If the same ratio applies for young children it can be assessed that the bioavailability of budesonide inhaled from a Nebuhaler will be around 55/90 $\times 25 \%=15 \%$ of the nominal dose, that is $40 \%$ lower than for schoolchildren. When such assessments are made it should be remembered that young children are more prone to inhale through the nose than older children. ${ }^{3}$ This may affect the clinical effect as well as the systemic effect of the inhaler but probably to the same extent for all the inhalers studied.

The total dead space added to the inhalers in the study was $45 \mathrm{ml}$. Although this volume was the same for all devices, it caused a higher percentage increase in volume for the low volume spacers than for the Nebuhaler. However, the volume inhaled by the children during 30 seconds by far exceeded this volume plus the volume of the spacer. Therefore, we do not believe that this increase in volume has influenced the results to any clinically important extent. The rather high minute ventilation probably also explains why spacer volume did not influence the dose to the patient. In an earlier study we found that attachment of a filter did not significantly affect the breathing pattern of the children. So it is most unlikely that the filter caused the high minute ventilation in the present study.

The information derived in our study applies to children studied awake. The results might be different if the treatment had been given during sleep as it is not known if the minute ventilation would increase to the same extent under such circumstances.

The finding that the Nebuhaler delivered more drug to the patient than the Aerochamber is in good agreement with the results in a recent in vitro study, which found that drug delivery was greatest from the Nebuhaler at tidal volumes similar to those measured in the present study. ${ }^{4}$ Only at six breaths of tidal volumes around $25 \mathrm{ml}$ did the Aerochamber enhance drug delivery as compared with the Nebuhaler. ${ }^{4}$ The clinical effect of the two inhalers has not been compared. However, in schoolchildren the Aerochamber resulted in less bronchodilatation than the Inspirease spacer, ${ }^{56}$ probably as a result of the lower output from the Aerochamber.

Apart from measuring the mean dose deposited on the filter it is also important to evaluate the dose range for each inhaler as this gives some idea about the reproducibility of the dose delivered to the patient. A 3.5-fold variation in dose was seen for the Aerochamber whereas a 2-fold variation was observed for the two other inhalers, indicating that these would deliver a more consistent dose to the patient. However, further studies on the intraindividual variation in dose are needed before firm conclusions can be made. In the present study we evaluated patients who had never used a spacer device before. Furthermore, it is possible that training will reduce the variation in dose delivery.

The results of comparative studies in new users of inhalation devices may be influenced by the experience of the investigator in the use of the various inhalers. In our clinic about $50 \%$ of young children normally use an Aerochamber, $45 \%$ a Nebuhaler, and about $5 \%$ a Babyspacer. So a negative effect in disfavour of the Babyspacer results cannot be excluded. However, differences in experience could not explain the poorer results after Aerochamber inhalations. Differences in design, plastic material, and valve system may all have caused a higher retainment of drug in the Aerochamber. The higher variation in dose is most likely to be due to differences in valve system.

With these reservations we think that the study provides some useful information about inhalation treatment in young children with asthma. The larger variation in dose and the lower dose delivered from the Aerochamber suggest that this device is not (as we believed before the study) preferable to the two other spacers in the treatment of very young children (older than one year). Furthermore, the finding that children tend to increase their minute ventilation when a facemask is applied to their face, suggests that spacer volume is not so critical as normally believed. This observation means that results from studies that assess the effect of an inhaler by setting a respirator to mimic the tidal breathing of a young child should be interpreted with some caution if this factor is not taken into account.

1 Lödrup Carlsen KC, Nikander K, Carlsen KH. How much nebulised budesonide reaches infants and toddlers? Arch nebulised budesonide reaches

2 Pedersen S, Steffensen G, Ekman I. Pharmacokinetics of budesonide in children with asthma. Eur $\mathcal{f}$ Clin Pharmacol 1987; 31: 579-82.

3 Pedersen $S$, Østergaard PA. Nasal inhalation as a cause of inefficient pulmonal aerosol inhalation technique in children. Allergy 1983; 38: 191-4.

4 Everard ML, Clark AR, Milner AD. Drug delivery from holding chambers with attached facemask. Arch Dis Child 1992; 67: 580-5.

5 Crimi N, Palermo F, Cacopardo B, et al. Bronchodilator effect of Aerochamber and Inspirease in comparison with metered dose inhaler. Eur $\mathcal{F}$ Respir Dis 1987; 71: 153-7.

6 Lee H, Evans HE. Evaluation of inhalation aids of metered dose inhalers in asthmatic children. Chest 1987; 91: 366-9.

\section{Commentary}

Although at first reading, one might be forgiven for thinking that this is just another study of a few of the potentially infinite combinations of puffers, spacers, facemasks and young children, it does have one important feature that distinguishes it from many other studies: the measurements are clinically relevant, albeit only under the precise conditions which applied during the study.

When one considers the huge effort that has gone into the development and marketing of topical asthma drugs, the trivial investment that has gone into the development of therapeutic devices for preschool children seems almost negligent. Pedersen has been almost alone over the last 12 years in showing, alas often in retrospect, the limitations of the devices which have been 'handed down' to young children.

Each aspect of aerosol administration by metered dose inhaler (MDI), spacer, and facemask needs careful study: the size, shape, 